\title{
Biomechanical evaluation of swing-through crutch gait in patients with lower extremity injury
}

\author{
AGATA RZEPNICKA ${ }^{1}$, JAROSŁAW KABACIŃSKI ${ }^{1 *}$, MiCHAŁ MURAWA ${ }^{1}$, ANNA FRYZOWICZ ${ }^{1}$, \\ MAŁGORZATA SYCZEWSKA ${ }^{2}$, LECHOSŁAW BOGDAN DWORAK ${ }^{3}$ \\ ${ }^{1}$ Department of Biomechanics, Poznan University of Physical Education, Poznań, Poland. \\ ${ }^{2}$ Kinesiology Lab, The Children's Memorial Health Institute, Warsaw, Poland. \\ ${ }^{3}$ The President Stanisław Wojciechowski State University of Applied Sciences in Kalisz, Kalisz, Poland.
}

\begin{abstract}
Purpose: The main purpose was to determine the values of spatio-temporal parameters and ground reaction forces during the swingthrough crutch gait. Methods: Eighteen male patients with unilateral injury within the foot, ankle or shank (age: $25.4 \pm 7.7$ years, body height: $1.79 \pm 0.06 \mathrm{~m}$, body mass: $76.1 \pm 11.5 \mathrm{~kg}$ ) participated in the study. In the experiment, 6-camera optoelectronic motion capture system and force platform were used. The measurements of spatio-temporal parameters and ground reaction force (GRF) were performed for uninjured lower extremity (ULE), crutch on the ULE side (CrU) and crutch on the injured lower extremity side (CrI). Results: Analysis demonstrated a significantly longer stance phase and a significantly shorter swing phase for ULE than crutches $(p<0.05)$, and a significantly longer first, compared to the second, double support phase $(p<0.05)$. Comparisons showed also significantly higher maximum values of the vertical GRF and extreme values of the horizontal antero-posterior GRF for ULE than crutches $(p<0.05)$ as well as for $\mathrm{CrI}$ than $\mathrm{CrU}(p<0.05)$. Conclusions: The present study provides biomechanical data related to the spatio-temporal parameters and GRFs for the swing-through crutch gait in patients with the lower extremity injuries.
\end{abstract}

Key words: swing-through gait, motion capture, spatiotemporal parameters, ground reaction force, lower extremity injury

\section{Introduction}

Swing-through gait is a symmetrical crutch gait characterized by alternating support first on the crutches and then on the feet [4], [18]. This gait type includes, successively, such phases as: the first double support (both crutches before the feet), crutch stance and lower extremity swing, the second double support (both crutches behind the feet), and crutch swing and lower extremity stance [4], [6]. Swing-through gait demands endurance and high muscles strength of upper extremities and trunk as well as good sense of balance [1], [4], [18], [20]. Long-term usage of this crutch gait can strengthen the muscles but it may also lead to overloads and injuries in upper extremities' joints or other structures [2].
Swing-through gait is used mainly by people after lower extremity (LE) amputation or paralysis, big plaster cast, and whenever weight bearing on injured LE is contraindicated [14]. Patients with LE injuries often choose this crutch gait due to the relatively high movement velocity. However, they has to adopt to unusual movement patterns and loads being transferred by carrying all body weight on arms, resigning from alternate pelvic movements [1], [2], [20].

Among the different types of crutch gait, during the swing-through gait the greatest loads are generated, originating from initial contact of the heel with the ground and swinging movement of the body supported on crutches [4], [9]. Several studies analysed external loads based on the values of the vertical ground reaction force (GRF) in healthy people simulating this type of gait [4], [6], [9], [12], [17]. Com-

\footnotetext{
* Corresponding author: Jarosław Kabaciński, Department of Biomechanics, Poznan University of Physical Education, ul. Królowej Jadwigi 27/39, 61-871, Poznań, Poland. Phone: +48-61-8355392, e-mail: biomechanika@awf.poznan.pl

Received: December 16th, 2019

Accepted for publication: March 3rd, 2020
} 
pared to the normal gait, Dworak et al. [4], Goh et al. [6], Nowotny-Czupryna [12] and Stallard et al. [17] found a higher maximal vertical GRF acting on the LE during swing-through gait.

Some investigators analysed temporal parameters for the swing-through gait. Goh et al. [6] reported temporal gait data for the stance phase and swing phase in healthy physical therapists who simulated swing-through axillary crutch gait. In turn, Rovick and Childress [13] determined magnitude of the baseline temporal parameters such as average velocity and relative time of the swing-through forearm crutch gait in one patient with spinal cord injury.

Previous available studies related to the swing-through gait mainly include individuals simulating this crutch gait. However, especially important is the comparative biomechanical analysis of the swing-through gait in injured people. Therefore, the main purpose of the study was to determine the values of spatio-temporal parameters and GRF for lower extremity and crutches during the swing-through crutch gait in patients with unilateral injury of the distal part of LE.

\section{Materials and methods}

\subsection{Participants}

A total of 18 male patients with unilateral injury in foot, ankle or shank area participated in the study.
Characteristics of the patients are presented in Table 1. Moreover, inclusion criteria were as follows: male, unilateral injury of distal part of LE, no other comorbidities, no serious orthopedic problems in medical history, fluent crutch walk. The patients also completed a questionnaire about causes of trauma, their experience with crutches and ailments resulting from this type of locomotion.

Table 1. Characteristics of the patients (mean \pm SD)

\begin{tabular}{|l|c|}
\hline \multicolumn{1}{|c|}{ Characteristic } & Mean \pm SD \\
\hline Age [years] & $25.4 \pm 7.7$ \\
\hline Body height $[\mathrm{m}]$ & $1.79 \pm 0.06$ \\
\hline Body mass $[\mathrm{kg}]$ & $76.1 \pm 11.5$ \\
\hline BMI $\left[\mathrm{kg} \cdot \mathrm{m}^{-2}\right]$ & $23.7 \pm 2.7$ \\
\hline Crutches $[$ days] & $23.0 \pm 16.5$ \\
\hline
\end{tabular}

BMI - body mass index, SD - standard deviation.

\subsection{Data collection}

The experimental protocol assumed: repetitive self-selected and comparable walking velocity, painless walking, central set of supporting LE or one of the crutches on the force platform, assumption that the movement of upper extremities with crutches was perfectly synchronized motion. The measurements of the kinematic parameters and ground reaction force (GRF) during the swing-through crutch gait were performed for uninjured lower extremity (ULE) and
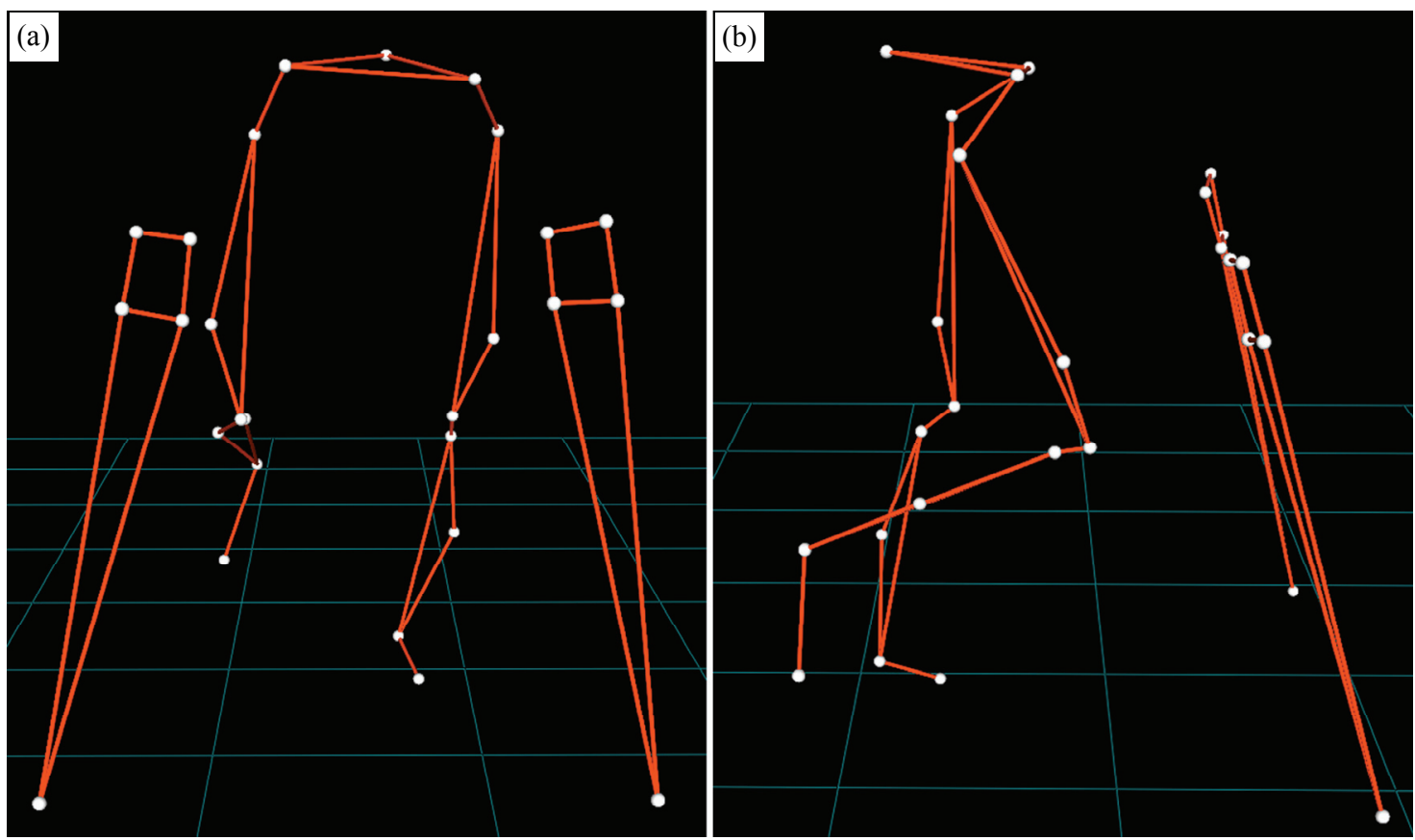

Fig. 1. The 27-markers biomechanical model during the swing-through gait of patient: (a) frontal plane, (b) sagittal plane 
crutches on the ULE side (CrU) and injured lower extremity (ILE) side (CrI). The swing-through crutch gait cycle (GC) was determined from the first to the second crutch strike and included successively the phases: (1) first double support of the crutches and ULE (crutches before the foot), (2) crutch stance and ULE swing, (3) second double support of the crutches and ULE (crutches behind the foot), and (4) crutch swing and ULE stance.

The 6-camera motion capture system $200 \mathrm{~Hz}$ (Smart-D, BTS Bioengineering, Milan, Italy) synchronized with one stationary force platform $800 \mathrm{~Hz}$ (BP400600, AMTI, Massachusetts, USA) were used. The values of the kinematic parameters and vertical component $(v)$, horizontal antero-posterior component $(y)$ and horizontal latero-medial component $(x)$ of GRF were collected using the BTS Smart Capture. According to Helen-Hayes Davis protocol, 27 special spherical markers (diameter of $20 \mathrm{~mm}$ ) reflecting infrared rays were placed within the pelvic girdle and lower extremities [3], [19]. Simple Davis protocol has been extended by 10 real markers placed on the crutches ( 5 on the $\mathrm{CrU}$ and 5 on the CrI) to analyse the displacements and the gait cycle phases (Fig. 1). Markers were tracked using the BTS Smart Tracker software.

Testing was preceded by several trials of the swingthrough gait on the measurement path to familiarize the participant with the experimental procedures. The measuring session consisted of the three series: the first series (ULE on the platform), the second series (CrU on the platform) and the third series (CrI on the platform), respectively. Each patient performed a dozen trials, however, for further analysis were taken data from 3 trials for ULE, 3 trials for CrU and 3 trials for CrI. Data were analysed using the BTS Smart Analyzer software.

\subsection{Data analysis}

The analysis included a) spatio-temporal parameters: stride length $(l)$ between the first and second crutch strike, gait velocity $(v)$ for stride length, width between the crutches $(w)$, stance phase time $\left(t_{S t}\right)$, swing phase time $\left(t_{S w}\right)$, first double support phase time $\left(t_{D b 1}\right)$ between the crutch strike and toe-off, second double support phase time $\left(t_{D b 2}\right)$ between the heel strike and crutch-off, b) time to maximum vGRF $\left(t_{\mathrm{vGRF}}\right)$ and maximal values of the vGRF, yGRF and xGRF for ULE and for crutches. The GRFs values were normalized to patients body weight (BW) and $100 \%$ of GC. Results of these variables for 54 trials were submitted to the further analysis.

\subsection{Statistical analysis}

Statistical analysis was performed using the Statistica 13.1 software (Statsoft Inc., Tulsa, Oklahoma, USA). Mean \pm standard deviation of the analysed variables were calculated. The distribution of the data was verified via the Shapiro-Wilk test. The paired samples $t$-test and non-parametric Wilcoxon signed-rank test were used for the comparisons between the phases, between the crutches, and between LE and crutches. Statistical significance for all analyses was defined by $p<0.05$.

\section{Results}

\subsection{Survey}

The survey showed that every participant was freely walking using the swing-through gait for minimum 7 days before participating in laboratory measurements. A significant proportion of participants (12 patients) suffered from ankle injury. Most of the injuries were the result of physical activity (15 patients) or accidental falls ( 3 patients). As many as 16 of subjects complained about hands and wrists soreness. They suffered from the shoulder girdle and free LE aches especially after passing a greater distance. In addition, they reported pain in the back and hip region - on the ULE side.

\subsection{Spatio-temporal parameters}

The values of spatio-temporal parameters for the swing-through crutch gait are presented in Table 2 $(l, v, w)$ and in Fig. 2a $\left(t_{S t}, t_{S w}, t_{D b 1}\right.$ and $\left.t_{D b 2}\right)$. Analysis showed a significantly longer stance phase for ULE ( $69 \%$ of GC) than crutches (55\% of GC) and a significantly shorter swing phase for ULE ( $31 \%$ of GC) than crutches $(45 \%$ of GC) $(p<0.05)$. In addition, significantly longer the $t_{D b 1}\left(13 \%\right.$ of GC) than the $t_{D b 2}(8 \%$ of GC) were found $(p<0.05)$.

Table 2. The values of selected spatio-temporal parameters (mean $\pm \mathrm{SD})$

\begin{tabular}{|l|c|}
\hline \multicolumn{1}{|c|}{ Variable } & Mean $\pm \mathrm{SD}$ \\
\hline$l[\mathrm{~m}]$ & $1.20 \pm 0.11$ \\
\hline$v\left[\mathrm{~m} \cdot \mathrm{s}^{-1}\right]$ & $0.78 \pm 0.10$ \\
\hline$w[\mathrm{~m}]$ & $0.8 \pm 0.1$ \\
\hline
\end{tabular}

$\mathrm{SD}$ - standard deviation, GC - swing-through gait cycle, $l$ - stride length, $v$ - gait velocity, $w$ - width between the crutches. 

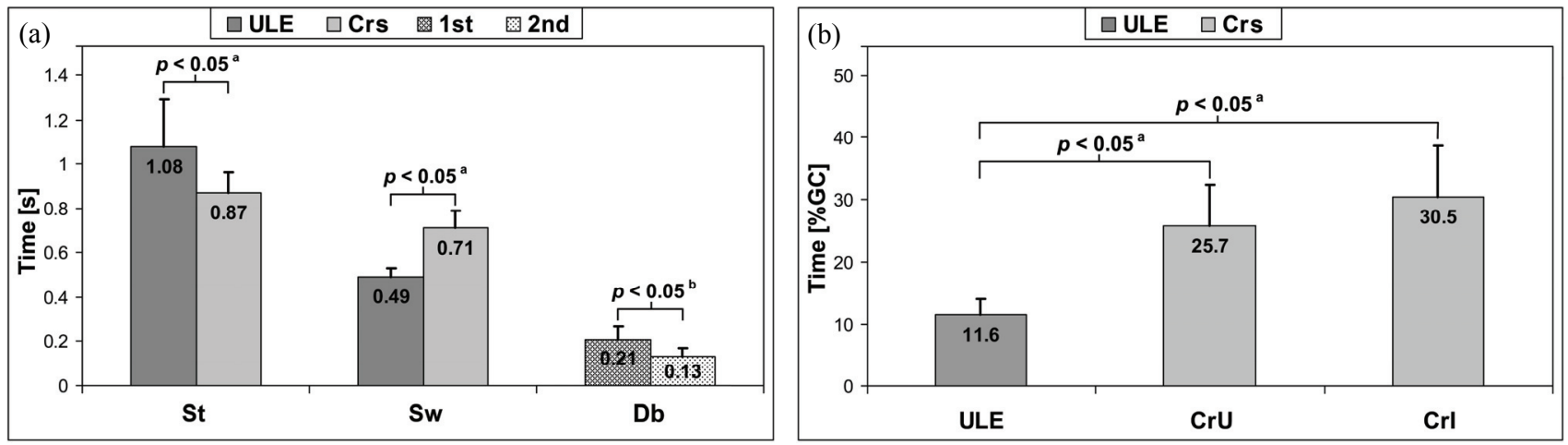

Fig. 2. Results of: a) time of the stance phase (St) and swing phase (Sw) for the uninjured lower extremity (ULE) and crutches (Crs), and 1st and 2nd double support phase (Db), b) time to develop maximal vGRF

during the swing-through gait cycle (GC) for ULE, crutch on the ULE side (CrU) and crutch on the ILE side (CrI):

(a) significance between the lower extremity and crutches (Wilcoxon test, $p<0.05$ ),

(b) significance between the double support phases (Wilcoxon test, $p<0.05$ )

\subsection{Ground reaction forces}

The GRF vs. GC curves for ULE, CrU and CrI during the swing-through crutch gait are shown in Fig. 3. The values of the $v \mathrm{GRF}, y \mathrm{GRF}$ and $x \mathrm{GRF}$ for lower extremity and crutches are presented in Table 3 . The analysis demonstrated significantly higher maximum values of the $v \mathrm{GRF}$ and $y \mathrm{GRF}$, and minimum values of the $y \mathrm{GRF}$ for the ULE than crutches as well as for the $\mathrm{CrI}$ than $\mathrm{CrU}(p<0.05)$. In the case of maximal $x \mathrm{GRF}$, no significant differences between the ULE and crutches independently ( $p=0.134)$ and between the $\mathrm{CrU}$ and $\mathrm{CrI}(p=0.206)$ as well as significantly higher summed values for both crutches compared to the ULE were found. Moreover, maximum values of the $v$ GRF were obtained after a longer $t_{v \mathrm{GRF}}$ for $\mathrm{CrU}$ and CrI than for ULE $(p<0.05)$ (Fig. 2b).
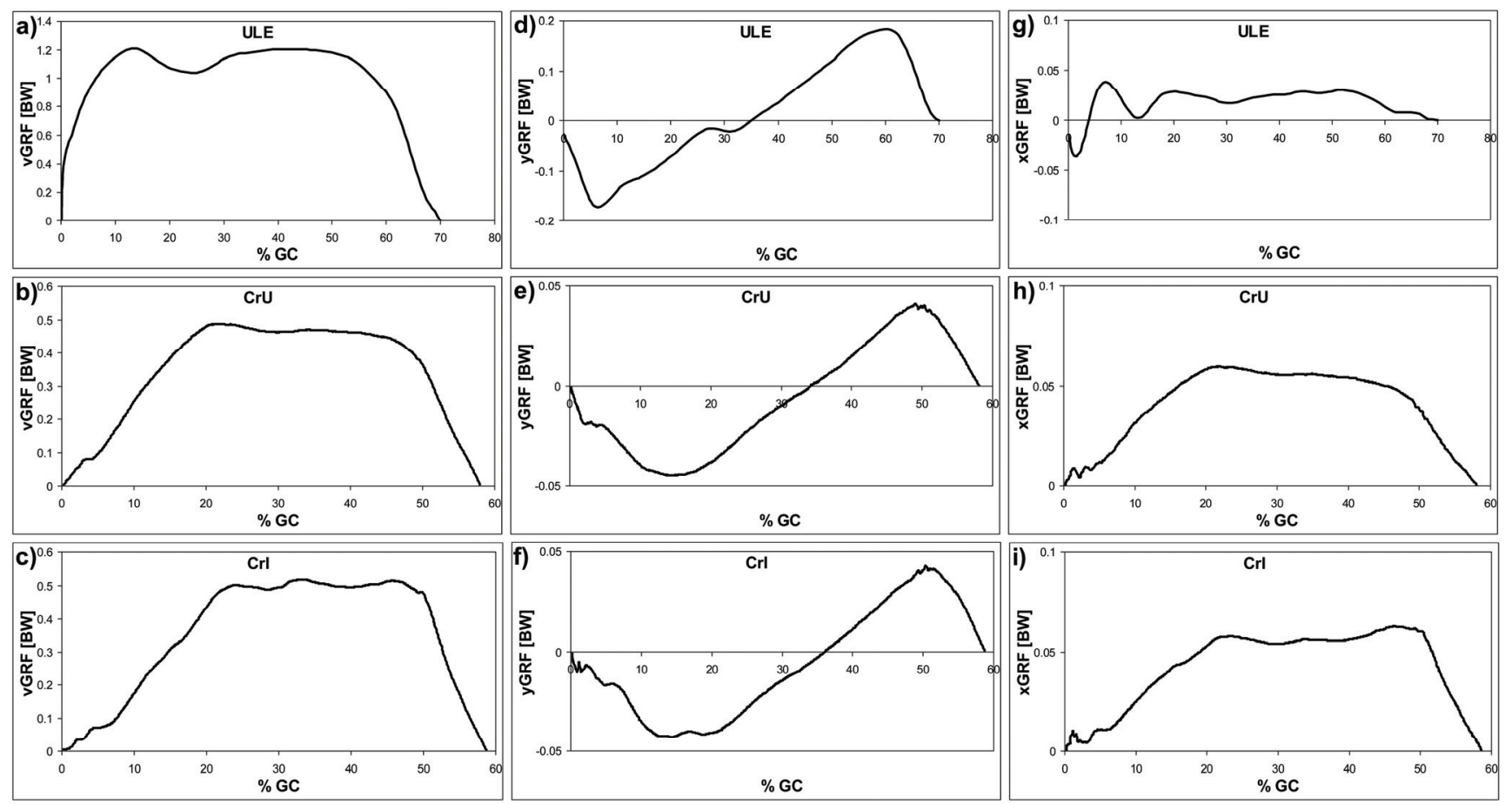

Fig. 3. Curves of the $v$ GRF (vertical ground reaction force) ((a), (b), (c)), $y$ GRF (horizontal antero-posterior component of ground reaction force) ((d), (e), (f)) and $x \mathrm{GRF}$ (horizontal latero-medial component of ground reaction force) ((g), (h), (i)) vs. swing-through crutch gait cycle (GC) during a representative trial 
Table 3. Mean $\pm \mathrm{SD}$ of GRFs during swing-through crutch gait and $p$-values

\begin{tabular}{|c|c|c|c|c|c|c|}
\hline \multirow[b]{2}{*}{ Variable } & \multirow{2}{*}{$\begin{array}{c}\text { Lower extremity } \\
\text { ULE }\end{array}$} & \multicolumn{2}{|c|}{ Crutches } & \multicolumn{3}{|c|}{$p$-values } \\
\hline & & $\mathrm{CrU}$ & $\mathrm{CrI}$ & $\begin{array}{l}\text { ULE vs. } \\
\text { CrU, CrI }\end{array}$ & $\begin{array}{l}\text { ULE vs. } \\
\text { CrU + CrI }\end{array}$ & CrU vs. $\mathrm{CrI}$ \\
\hline Max. $v$ GRF [BW] & $1.17 \pm 0.11$ & $0.49 \pm 0.04$ & $0.52 \pm 0.03$ & $<0.05^{\mathrm{a}}$ & $<0.05^{\mathrm{a}}$ & 0.098 \\
\hline Min. $y$ GRF $[\mathrm{BW}]$ & $0.19 \pm 0.05$ & $0.04 \pm 0.01$ & $0.05 \pm 0.02$ & $<0.05^{\mathrm{a}}$ & $<0.05^{\text {a }}$ & $<0.05^{\mathrm{c}}$ \\
\hline Max. $y$ GRF $[\mathrm{BW}]$ & $0.17 \pm 0.04$ & $0.04 \pm 0.01$ & $0.05 \pm 0.02$ & $<0.05^{\mathrm{a}}$ & $<0.05^{\mathrm{a}}$ & $<0.05^{\mathrm{c}}$ \\
\hline Max. $x$ GRF $[\mathrm{BW}]$ & $0.04 \pm 0.01$ & $0.04 \pm 0.01$ & $0.04 \pm 0.01$ & 0.134 & $<0.05^{\mathrm{a}}$ & 0.206 \\
\hline
\end{tabular}

$\mathrm{SD}$ - standard deviation, max $v \mathrm{GRF}$ - maximum value of the vertical ground reaction force, min $y \mathrm{GRF}$ - minimum value of the horizontal antero-posterior component of ground reaction force, max $y \mathrm{GRF}$ - maximum value of the horizontal antero-posterior component of ground reaction force, max $x \mathrm{GRF}$ - maximum value of the horizontal latero-medial component of ground reaction force, ULE - uninvolved lower extremity, CrU - crutch on the uninjured lower extremity side, CrI - crutch on the injured lower extremity side, ${ }^{\mathrm{a}}$ - significance between the lower extremity and crutches $(t$-test, $p<0.05),{ }^{\mathrm{c}}-$ significance between the crutches ( $t$-test, $p<0.05)$.

\section{Discussion}

The present study determined the values of selected biomechanical parameters during the swingthrough crutch gait in patients with the lower extremity injury. The analysis demonstrated that durations of the stance and swing phases varied considerably between the ULE and crutches. The stance phase lasted much longer for ULE ( $69 \%$ of GC) than for crutches ( $55 \%$ of GC), whereas time of the swing phase was significantly shorter for ULE ( $31 \%$ of GC) than for crutches ( $45 \%$ of GC). This trend for the swing-through gait can be associated with the greater certainty, stability and less effort of patients during stance on ULE than during the support on crutches. Compared to the normal gait data [19], these findings indicated a longer stance phase (by approx. 9\%) and a shorter swing phase (by approx. 9\%). Considering the swing-through gait, Rovick and Childress [13] reported $52 \%$ of GC (stance phase) and $48 \%$ of GC (swing phase) in paraplegic subject. In turn, results of Goh et al. [6] showed $72 \%$ of GC (stance phase) and $28 \%$ of GC (swing phase) for the simulated swing-through axillary gait.

The relatively long time of stance phase has contributed to lowering of velocity of subjects. The slower gait may be caused by the natural increased care of patients after injury compared to the individuals simulating the swing-through gait [6], [12], [16]. In regard to the normal gait, the swing-through gait in patients can be considered slow [10]. Furthermore, the higher average velocity was registered by Rovick and Childress [13] $\left(0.9 \mathrm{~m} \cdot \mathrm{s}^{-1}\right)$ and Stallard et al. [17] $\left(1.44 \mathrm{~m} \cdot \mathrm{s}^{-1}\right)$ for the swing-through gait.

The study also demonstrated that time of the first double support was significantly longer than the second double support (by approx. $38 \%$ ). The difference between both phases is related to the observation that foot detachment comes no sooner than the crutches were stabilized which required longer time. In comparison, results of the first double support phase time (12\% of GC) and the second double support phase time ( $10 \%$ of GC) reported by Rovick and Childress [13] showed a smaller difference (approx. 17\%). In contrast, Stallard et al. [17] obtained shorter time of the first double support phase time ( $6.9 \%$ of GC) and the second double-support phase (3.6\% of GC), and a greater difference (approx. 48\%).

The ULE loading determined by the maximal $\nu$ GRF $(1.17 \mathrm{BW})$ does not exceed the normative maximal $v$ GRF values for normal gait [5], [7], [11], [19], [22]. This study included only patients with actual LE injury, therefore amortization, slower gait and increased caution resulted in lower $v$ GRF. Other investigations revealed higher $v \mathrm{GRF}$ values for the LE, i.e., $1.28 \mathrm{BW}$ [6] and 1.32 BW [17], however, in people simulating the swing-through gait. In turn, lower $v$ GRF values were registered during the three-point crutch gait involved patients after total hip arthroplasty (1.04 BW for mid stance and $1.08 \mathrm{BW}$ for terminal stance) [8]. In the case of the horizontal antero-posterior GRF, similar results of the $y \mathrm{GRF}$ between the swing-through crutch gait and normal gait were observed [15], [22]. Furthermore, Stallard et al. [17] found greater $y \mathrm{GRF}$ values $(0.27 \mathrm{BW}$ - braking peak and $0.35 \mathrm{BW}$ - propulsive peak) compared to the normal gait. In addition, it's worth adding that Westerhoff et al. [21] examined joint loads during crutch-assisted walking in six patients with osteoarthritis of the shoulder and functional rotator cuff and received values of the shoulder joint forces equal 0.97-1.72 BW (elbow crutches) and 0.80-1.28 BW (forearm crutches).

Comparable analysis demonstrated significantly greater $v$ GRF values for lower extremity than for crutches independently (more than 50\%) and simulta- 
neously (approx. 15\%). Although, higher $v$ GRF values for both crutches were expected, the results revealed increased external loads on the lower extremity during the stance phase of swing-through gait. Considering to the horizontal components, significantly greater maximum and minimum $y \mathrm{GRF}$ values for ULE than crutches and significantly lower $x$ GRF values for ULE compared to both crutches simultaneously were recorded. In turn, comparisons of the GRFs between the crutches showed no significant differences in the maximum $v$ GRF and $x$ GRF values (below $6 \%$ ) and significantly higher extreme $y \mathrm{GRF}$ values for CrI than $\mathrm{CrU}$ (approx. 20\%). However, the percentage differences in the extreme GRF values between the crutches accounted for only approx. 1\% of BW (horizontal) and $3 \%$ of BW (vertical). The magnitude of horizontal components of GRFs for crutches depends on the angle of crutch inclination in the sagittal plane (yGRF) and frontal plane ( $x \mathrm{GRF})$. In the case of $v \mathrm{GRF}$, similar force results for both crutches can be attributed to the bilateral load pattern associated with the simultaneous crutches pressure.

This study also compared time to maximum $v$ GRF values between the lower extremity and crutches. The maximum $v$ GRF for ULE was developed after a shorter time (approx. 12\% of GC) than for $\mathrm{CrU}$ (approx. 30\% of GC) and CrI (approx. 25\% of GC). During the crutch initial contact, inclination angle of crutch is the smallest and in this phase the lowest $v$ GRF values were recorded. Thus, the highest $v$ GRF for $\mathrm{CrU}$ was generated after a longer time compared to the ULE until the inclination angle of crutch was close to $90^{\circ}$.

\section{Conclusions}

Findings revealed significant differences in the time of stance, swing and double support phases, as well as $v$ GRF and $y$ GRF values. The $v$ GRF results indicated increased external loads for the lower extremity, compared to crutches, and for the crutch on the injured lower extremity, compared to the opposite crutch. In addition, results of spatio-temporal and dynamic parameters were confronted to literature data for simulated swing-through crutch gait and normal gait. Patients in this study generated smaller $v$ GRF for the lower extremity than participants simulating the swing-through crutch gait. The analysis also showed a longer stance phase and a shorter swing phase as well as lower $v$ GRF values for the uninjured lower extremity compared to the normative data of normal gait. In sum- mary, this study reported biomechanical data on the spatio-temporal structure and loading pattern for the swing-through crutch gait in patients with the lower extremity injuries.

\section{Acknowledgements}

This study was financed by PhD grant No. N N404 271540 of the Polish Ministry of Science and Higher Education. The authors would like to thank all participating patients.

\section{References}

[1] BATENi H., MaKi B.E., Assistive devices for balance and mobility: benefits, demands and adverse consequences, Arch. Phys. Med. Rehabil., 2005, 86(1), 134-145.

[2] Carpentier C., Font-Llagunes J.M., KöVecses J., Dynamics and energetics of impacts in crutch walking, J. Appl. Biomech., 2010, 26 (4), 473-483.

[3] Davis R.B., Ounpuu S., Tyburski D., Gage J.R., A gait analysis data collection and reduction technique, Hum. Mov. Sci., 1991, 10 (5), 575-587.

[4] Dworak L.B., Rzepnicka A., Murawa M., Maczynski J., BuszKo P., Swing-through gait from the perspective of biomechanics and kinesiology. Critical analysis of the current state of knowledge and the idea behind the research, Pol. Orthop. Traumatol., 2010, 75 (6), 392-398.

[5] Fryzowicz A., Murawa M., Kabaciński J., RzePnicka A., DWORAK L.B., Reference values of spatiotemporal parameters, joint angles, ground reaction forces, and plantar pressure distribution during normal gait in young women, Acta Bioeng. Biomech., 2018, 20 (1), 49-57.

[6] GoH J.C., TоH S.L., Bose K., Biomechanical study on axillary crutches during single-leg swing-through gait, Prosthet. Orthot. Int., 1986, 10 (2), 89-95.

[7] Keller T.S., Weisberger A.M., Ray J.L., Hasan S.S., SHIAVI R.G., SPENGLER D.M, Relationship between vertical ground reaction force and speed during walking, slow jogging, and running, Clin. Biomech., 1996, 11 (5), 253-259.

[8] Murawa M., Dworak L.B., KabaciŃSKi J., SyczewsKa M., RZEPNICKA A., Dynamic parameters of three-point crutch gait in female patients after total hip arthroplasty, Acta Bioeng. Biomech., 2016, 18 (2), 131-135.

[9] Nowotny-CZupryna O., Nowotny J., SOŁtys J., BrzęK A., CZupryna K., Dabrowska J., Possibilities of one leg relief while walking on crutches, Physiotherapy, 2005, 13 (3), 48-56.

[10] ÖBERG T., KARSZNIA A., ÖBERG K., Joint angle parameters in gait: reference data for normal subjects, 10-79 years of age, J. Rehab. Res. Dev., 1994, 31 (3), 199-213.

[11] Perry J., Burnfield J.M., Gait analysis: normal and pathological function, Slack Incorporated, 2010.

[12] Reisman M., Burdett R.G., SimOn S.R., Norkin C., Elbow moment and forces at the hands during swing-through axillary crutch gait, Phys. Ther., 1985, 65 (5), 601-605.

[13] Rovick J.S., ChILDRess D.S., Pendular model of paraplegic swing-through crutch ambulation, J. Rehabil. Res. Dev., 1988, 25 (4), 1-16.

[14] Shoup T.E., Fletcher L.S., Merrill B.R., Biomechanics of crutch locomotion, J. Biomech., 1974, 7 (1), 11-19. 
[15] Simoneau G.G., Kinesiology of waking, [in:] D.A. Neumann (Ed.), Kinesiology of the musculoskeletal system: foundations for rehabilitation, Mosby/Elsevier, 2010.

[16] Slavens B.A., Sturm P.F., Harris G.F., Upper extremity inverse dynamics model for crutch-assisted gait assessment, J. Biomech., 2010, 43 (10), 2026-2031.

[17] Stallard J., Dounis E., Major R.E., Rose G.K., One leg swing-through gait using two crutches, Acta Orthop. Scand., 1980, 51 (1), 71-77.

[18] Thys H., Willems P.A., SAels P., Energy cost, mechanical work and muscular efficiency in swing-through gait with elbow crutches, J. Biomech., 1996, 29 (11), 1473-1482.
[19] Vaughan C., Davis B., O'Connor J., Dynamics of human gait, Kiboho Publishers, 1992.

[20] Waters R.L., Mulroy S., The energy expenditure of normal and pathologic gait, Gait Posture, 1999, 9 (3), 207 $-231$.

[21] Westerhoff P., Graichen F., Bender A., Halder A., Beier A., ROHLMANN A., BERGMANN G., In vivo measurement of shoulder joint loads during walking with crutches, Clin. Biomech., 2012, 27 (7), 711-718.

[22] WINTER D.A., Biomechanics and motor control of human gait: normal, elderly and pathological, University of Waterloo Press, 1991. 